\section{DEFECT OF BDH2 CONTRIBUTES TO DNA HYPOMETHYLATION IN CD4+ T CELLS OF SYSTEMIC LUPUS ERYTHEMATOSUS}

M Zhao*, M Li, Q Lu. The Second Xiangya Hospital of Central South University, Department of Dermatology, Changsha, China

\subsection{6/lupus-2017-000215.336}

Background and aims A lot of evidences have confirmed that genomic DNA hypomethylation play an important role in the pathogenesis of systemic lupus erythematosus (SLE). However, the mechanism of DNA hypomethylation in lupus CD4+ T cells remain unclear. Previous study showed that depletion of the mammalian siderophore by inhibiting expression of $3-\mathrm{OH}$ butyrate dehydrogenase (BDH2) results in abnormal accumulation of intracellular iron and mitochondrial iron deficiency in cultured mammalian cells. In this study, we investigate whether $\mathrm{BDH} 2$ is involved in regulating DNA hypomethylation in CD4+ T cells of SLE.

Methods 20 SLE patients and 20 healthy controls were recruited. CD4 $+\mathrm{T}$ cells were isolated by magnetic beads. All patients fulfilled at least 4 of the SLE classification criteria of ACR. mRNA and protein levels were detected by real-time PCR and western blot. Global DNA methylation level was measured by Global DNA Methylation Assay-LINE-1 kit. CD4 $+\mathrm{T}$ cells were transfected by nucleofector.

Results Compared with normal controls, BDH2 mRNA and protein levels were decreased significantly in SLE CD4 $+\mathrm{T}$ cells, which are positively correlated with the global DNA methylation levels. Knockdown of $\mathrm{BDH} 2$ with siRNA in normal CD4+ T cells decreased the global DNA methylation level compared with negative control. In contrast, overexpressing $\mathrm{BDH} 2$ with expression plasmid can increase the global DNA methylation level in SLE CD4+ T cells.

Conclusions BDH2 expression was defect in CD4 $+\mathrm{T}$ cells of SLE patients, which contributes to the genomic DNA hypomethylation of CD4+ T cells in SLE patients.

\section{Innate immunity}

\section{THE SIGNIFICANT ROLE OF TOLL-LIKE RECEPTOR IN SYSTEMIC LUPUS ERYTHEMATOSUS}

FF Alkaff*, S Salamah, RA Nugraha, M Jonatan. Airlangga University, Faculty of Medicine, Surabaya, Indonesia

\subsection{6/lupus-2017-000215.337}

Background and aims Until nowadays, the exact aetiology of SLE (Systemic Lupus Erythematosus) is still unknown. It is currently accepted that there are several factors responsible for complex immunological disorders contributing to its development. Recent studies have shown that abnormal stimulation of innate immunity may have a great influence on the immunopathogenesis of SLE. Since TLRs (Toll-Like Receptors) are essential modulators of innate immune response, its role in SLE pathogenesis has raised great interest, particularly of those recognising nucleic acid, the main antigenic target in SLE.

Analyse the role of TLR in the pathogenesis of SLE.

Methods We look up for scienctific article comprehensively in Medline, Science Direct, PubMed, and Cochrane Database. We found 20 article based on bibliography and keywords from the database.
Results The expression of TLR7 and TLR9 in peripheral blood mononuclear cells is higher in SLE group compared to control group, while the expression of other TLR shows no difference. There is also a positive correlation between TLR9 expression and activity index and R-SLEDAI score, while TLR7 expression shows positive correlation with chronicity index. Anti-Sm autoantibodies is absent in TLR7 deficiency, while anti-dsDNA autoantibodies are absent in TLR9 deficiency. TLR7 also plays crucial role in B cell proliferation.

Conclusions TLR7 and TLR9 appears to play role in pathogenesis of SLE. However, more research need to be done to understand more about the role of TLR in pathogenesis of SLE.

\section{THE ROLE OF TLR8 IN LUPUS NEPHRITIS}

A Davidson*, M Woods, N Maria. Feinstein Institute for Medical Research, Autoimmune and musculoskeletal diseases, Manhasset, USA

\subsection{6/lupus-2017-000215.338}

Background and aims Lupus nephritis (LN) is a significant cause of morbidity and mortality. In recent systems biology work we found that renal macrophage functional pathways that link phagocytosis with activation of TLR pathways and disposal of excess cellular components are shared between mice and humans with lupus nephritis. Surprisingly we found that in two different lupus strains there is marked upregulation of renal TLR8 expression restricted to resident renal macrophages.

Methods Because mouse TLR8 does not recognise ssRNA, we generated NZW/B6.Yaa mice expressing a functional form of human TLR8 as a BAC transgene to examine the effect of human TLR8 on systemic immunity and renal inflammation. TLR8 mRNA was quantitated by qPCR. Male and female mice were followed clinically for up to one year and harvested for flow cytometry analysis of spleens and kidneys. 24week-old male mice were administered TL-506 subcutaneously for 4 weeks and harvested after 8 weeks.

Results A single dose of human TLR8 in NZW/B6.Yaa mice did not exacerbate disease or accelerate disease or change the immune phenotype of the spleens or kidneys. However administration of a TLR8 agonist to male NZW/B6.Yaa mice appeared to enhance germinal centre formation and plasma cell generation in transgenic mice.

Conclusions A single dose of human TLR8 is not sufficient to initiate or exacerbate lupus in NZW/B6.Yaa mice. Preliminary findings suggest that the mice may be hyperresponsive to a TLR8 agonist. Lupus mice with 2 copies of the human TLR8 transgene have been generated to determine whether an extra dose will affect lupus onset or phenotype.

\section{THE ROLE OF NEUTROPHILS IN ORGAN TISSUE DAMAGE IN SLE}

X Guo, GM Deng*. Nanjing Medical University, Key Laboratory of Antibody Techniques of Ministry of Health, Nanjing, China

\subsection{6/lupus-2017-000215.339}

Background and aims Systemic lupus erythematosus (SLE) is a chronic autoimmune disease characterised by high levels of autoantibodies and multi-organ damage. Neutrophils are the 